HETEROCYCLES, Vol. 65, No. 2, 2005, pp. 387 - 394

Received, 19th October, 2004, Accepted, 1st December, 2004, Published online, 3rd December, 2004

\title{
FLUORINATED HETEROCYCLIC COMPOUNDS.
}

\section{A PHOTOCHEMICAL APPROACH TO A SYNTHESIS OF POLYFLUOROARYL-1,2,4-TRIAZOLES}

\author{
Silvestre Buscemi,* Andrea Pace, Antonio Palumbo Piccionello, Ivana Pibiri, and \\ Nicolò Vivona \\ Dipartimento di Chimica Organica “E. Paternò”, Università degli Studi di \\ Palermo, Viale delle Scienze-Parco d'Orleans II, I-90128, Palermo, Italy \\ E-mail:sbuscemi@unipa.it
}

\begin{abstract}
The reaction of some fluorinated 1,2,4-oxadiazoles in the presence of methylamine or propylamine has been investigated. The irradiation in methanol or acetonitrile leads with acceptable yields to the corresponding fluorinated 1methyl- or 1-propyl-1,2,4-triazole.
\end{abstract}

\section{INTRODUCTION}

Fluorinated heterocycles represent an extremely interesting class of compounds, thanks to their possibile application in the fields of new materials, pharmaceuticals or agrochemicals. ${ }^{1,2}$ Their synthesis through direct fluorination or perfluoroalkylation could be quite complex, therefore the building block strategy often represents a more appropriate approach. ${ }^{1,2}$

Within the building block strategy, the use of molecular rearrangements of a suitable heterocyclic precursor has been exploited for the synthesis of several fluorinated heterocyclic systems. In particular,

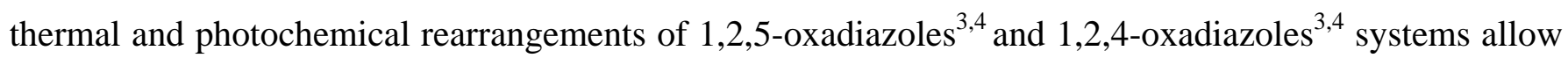
the synthesis of a variety of fluorinated heterocyclic compounds such as 1,2,4-oxadiazoles, ${ }^{5} 1,2,4$ triazoles, ${ }^{6}$ 1,3,4-oxadiazoles, ${ }^{6}$ and quinazolin-4-ones. ${ }^{7}$

Among the plethora of five-membered heterocycles, 1,2,4-triazoles showed various biological activities: as an example they have been used as cannabinoid receptor ligands ${ }^{8}$ or peptidase inhibitors, ${ }^{9}$ and some have been tested for the treatment of viral infections or cancer. ${ }^{10}$ More recently, fluorinated triazoles have been applied in the preparation of ionic liquids, ${ }^{11}$ in the treatment of neuropathic pain ${ }^{12}$ or as antifungal, ${ }^{13}$ and their syntheses have been subjected to several patents. ${ }^{14}$

For what concerns the synthetic aspects, fluorinated triazoles are not of easy preparation and some examples are present in the recent literature. ${ }^{11,15}$ In particular, perfluoroalkyl-1,2,4-triazoles can be also obtained by a thermal ANRORC reaction of 5-perfluoroalkyl-1,2,4-oxadiazoles with hydrazines ${ }^{16}$ or by a photochemical rearrangement of 3-methylamino-5-perfluoroalkyl-1,2,4-oxadiazoles. ${ }^{6}$ 
In the frame of our research on fluorinated heterocycles, we have widened the series of achievable fluorinated 1,2,4-triazoles and here we report a photochemical methodology for obtaining of polyfluoroaryl-1,2,4-triazoles.

\section{RESULTS AND DISCUSSION}

In this work, we exploited the well known photoreaction of the 1,2,4-oxadiazole system (1) which undergoes photolytic cleavage of the O-N bond producing a zwitterion or nitrene-like species (2). This species can easily be attacked by a nucleophile present in the photoreaction medium (a primary amine for example) to form open-chain intermediates (3) that will eventually cyclize into the final heterocyclic system (a $N$-alkyl-1,2,4-triazole) (Scheme 1). ${ }^{17}$

\section{Scheme 1}<smiles>[R]c1noc([R])n1</smiles>

1<smiles>[R]C(=[No])N=C([R])CC</smiles>

2

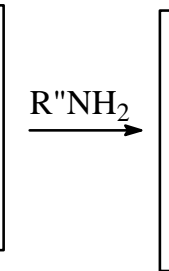<smiles>[R]N/N=C(\[R])N=C([R])O</smiles>

3<smiles>[R]c1nc([R])n([R7])n1</smiles>

4

The investigated 1,2,4-oxadiazoles [derivatives (5a-e)] are characterized by the presence of a pentafluorophenyl at C3 (5a) or a polyfluorophenyl moiety at the C5 of the heterocycle (5b-e). The use of the 5-pentafluorophenyl derivative has been avoided because of some inconvenient due to the peculiar reactivity of 5-pentafluorophenyl-1,2,4-oxadiazoles, that easily undergo nucleophilic aromatic substitution of the $p$-fluoro- substituent by the primary amine used in the reaction. ${ }^{5 b}$

Irradiations (at $\lambda=254 \mathrm{~nm}$ ) have been carried out in oxygenated methanol and in the presence of a large excess of methyl- or propylamine. The choice of the above conditions is a result of several experiments where the influence of the solvent, the amine concentration and oxygenation was investigated. In acetonitrile, for example, the formation of the desired triazoles (in lower yields than in methanol though) was accompained by a series of unidentified decomposition products. On the other hand, in methanol at least 3 additional competitive pathways can be identified (Scheme 2).

The formation of solvolysis products (10) is due to the nucleophilic attack of the methanol (that competes with the amine) on the photolytic intermediate (6). The use of a large excess of amine, therefore, will favor the formation of the intermediate (7) and drive the reaction towards the 1,2,4-triazoles (8) or (9). 
Scheme 2

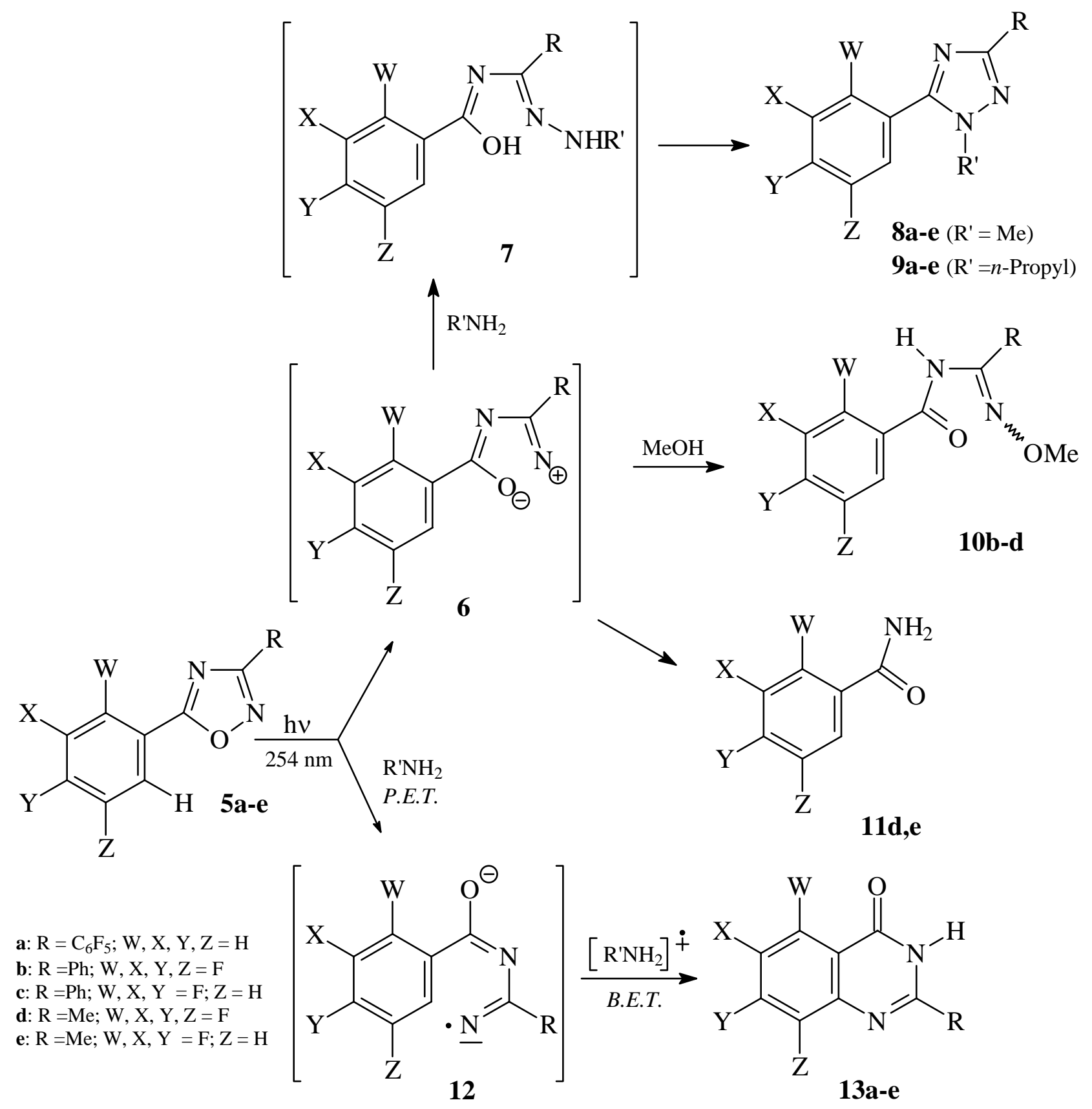

P.E.T. $=$ Photoinduced Electron Transfer

B.E.T. $=$ Back Electron Transfer

Unfortunately, the competing formation of the quinazolin-4-one (13), is also favored by the excess of the amine, since a photoinduced electron transfer $^{18}$ (from the amine to the excited oxadiazole) is responsible for the formation of the radical-anion (12) that leads to the quinazolin-4-one. This reaction can be partially quenched by oxygenating the solution prior to irradiation. Finally, the formation of amides (11) is due to a hydrolysis reaction of the photolytic intermediate (6). This has been observed only for 3methyl-1,2,4-oxadiazoles (5d,e), while the presence of an aryl moiety at C3 (either fluorinated or not) inhibits the hydrolytic process. 
The results of photoreaction are summarized in Table 1; physical and analytical data for the isolated products are reported in Table 2.

This methodology cannot be applied to perfluoroalkyl derivatives; hydrolytic processes are, infact, the main inconvenient in the irradiation of 3-perfluoroalkyloxadiazoles from which the corresponding triazole could not be isolated. At the same time, 5-perfluoroalkyloxadiazole (14) produces the perfluoroalkyl amide (17) as the main product, probably as a result of a preliminary nucleophilic attack at C5 of the oxadiazole, ${ }^{16,19}$ followed by photolytic cleavage (Scheme 3).

\section{Scheme 3}

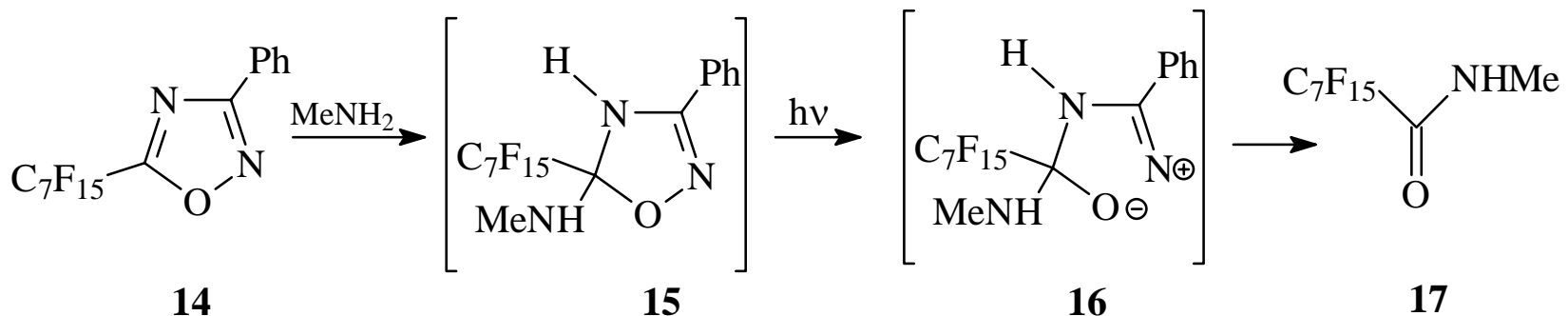

14

As a final comment, although yields are not excellent, this photochemical approach represents a useful and alternative strategy to obtain fluoroaryl-1,2,4-triazoles, whose preparation by conventional methods ${ }^{16,20}$ could be difficult.

Table 1. - Irradiations of compounds (5a-e) in the presence of primary amines.

\begin{tabular}{ccccccccccc}
\hline \hline \multicolumn{1}{c}{$\begin{array}{c}\text { Substrate } \\
\text { (\% Recovered) }\end{array}$} & Amine & \multicolumn{8}{c}{ Product Distribution } \\
\hline $\mathbf{5 a}$ & $(20)$ & $\mathrm{MeNH}_{2}$ & $\mathbf{8 a}$ & $(35)$ & $\mathbf{1 3 a}$ & $(20)$ & & & \\
$\mathbf{5 b}$ & $(25)$ & $\mathrm{MeNH}_{2}$ & $\mathbf{8 b}$ & $(37)$ & $\mathbf{1 3 b}$ & $(3)$ & $\mathbf{1 0 b}$ & $(10)$ & & \\
$5 \mathbf{c}$ & $(17)$ & $\mathrm{MeNH}_{2}$ & $\mathbf{8 c}$ & $(45)$ & $\mathbf{1 3 c}$ & $(2)$ & $\mathbf{1 0 c}$ & $(12)$ & & \\
$\mathbf{5 d}$ & $(16)$ & $\mathrm{MeNH}_{2}$ & $\mathbf{8 d}$ & $(35)$ & $\mathbf{1 3 d}$ & $(5)$ & $\mathbf{1 0 d}$ & $(15)$ & $\mathbf{1 1 d}$ & $(6)$ \\
$\mathbf{5 e}$ & $(15)$ & $\mathrm{MeNH}_{2}$ & $\mathbf{8 e}$ & $(46)$ & $\mathbf{1 3 e}$ & $(2)$ & & & $\mathbf{1 1 e}$ & $(15)$ \\
$\mathbf{5 a}$ & $(20)$ & $n-\mathrm{PrNH}_{2}$ & $\mathbf{9 a}$ & $(34)$ & $\mathbf{1 3 a}$ & $(22)$ & & & & \\
$\mathbf{5 b}$ & $(25)$ & $n-\mathrm{PrNH}_{2}$ & $\mathbf{9 b}$ & $(36)$ & $\mathbf{1 3 b}$ & $(3)$ & $\mathbf{1 0 b}$ & $(12)$ & & \\
$\mathbf{5 c}$ & $(20)$ & $n-\mathrm{PrNH}_{2}$ & $\mathbf{9 c}$ & $(43)$ & $\mathbf{1 3 c}$ & $(2)$ & $\mathbf{1 0 c}$ & $(10)$ & & \\
$\mathbf{5 d}$ & $(16)$ & $n-\mathrm{PrNH}_{2}$ & $\mathbf{9 d}$ & $(34)$ & $\mathbf{1 3 d}$ & $(5)$ & $\mathbf{1 0 d}$ & $(15)$ & $\mathbf{1 1 d}$ & $(6)$ \\
$\mathbf{5 e}$ & $(15)$ & $n-\mathrm{PrNH}_{2}$ & $\mathbf{9 e}$ & $(49)$ & $\mathbf{1 3 e}$ & $(2)$ & & & $\mathbf{1 1 e}$ & $(13)$ \\
\hline \hline
\end{tabular}




\section{EXPERIMENTAL}

General: Melting points were determined on a REICHART-THERMOVAR hot-stage apparatus and are uncorrected. IR spectra (Nujol) were determined with a PERKIN ELMER 257 instrument; ${ }^{1} \mathrm{H}-\mathrm{NMR}$ spectra were recorded on a BRUKER AC 250 E spectrometer, and GC/MS spectral determinations were carried out on a VARIAN STAR 3400 CX/SATURN 2000 system. Flash chromatography was performed by using silica gel (Merck, 0.040-0.063 mm) and mixtures of ethyl acetate and light petroleum (fraction boiling in the range $40-60^{\circ} \mathrm{C}$ ) in various ratios. Dry methanol (from Romil Pure Chemicals) was used as received.

Compounds (5a), ${ }^{21}$ (5b-e), ${ }^{7}$ (14), ${ }^{22}$ (used for irradiation) and $\left(\mathbf{1 3 a - e )}{ }^{7}\right.$ (11d), ${ }^{23}$ (17) ${ }^{24}$ (used for comparison) were prepared as reported. Compound (11e) was prepared by reaction between 2,3,4trifluorobenzoyl chloride and ammonia. Compound (11e) had mp $134-135^{\circ} \mathrm{C}$ (light petroleum), IR 3400, 3190, $1660 \mathrm{~cm}^{-1} ;{ }^{1} \mathrm{H}$ NMR (DMSO-d 6 ) $\delta$ 7.40-7.65 (m, 2H, ArF); 7.85 (s, 1H, NH); 7.95 (s, 1H, NH); MS m/z 175 ( $\left.\mathrm{M}^{+}, 100\right), 159$ (79), 131 (39), 81 (38). Anal. Calcd for $\mathrm{C}_{7} \mathrm{H}_{4} \mathrm{NOF}_{3}$ : C, 48.01; H, 2.30; N, 8.00. Found: C,47.90; H,2.20; N, 7.90.

\section{General Procedure for Photochemical Reactions}

Photochemical reactions were carried out by using a Rayonet RPR-100 photoreactor fitted with $16 \mathrm{Hg}$ lamps irradiating at $\lambda=254 \mathrm{~nm}$ (RPR-2537 $\AA$ ) (Quartz vessels) and equipped with a merry-go-round apparatus.

A solution of compound (5a-e) $(1.28 \mathrm{mmol})$ in dry methanol $(400 \mathrm{~mL})$, was partitioned into nine pyrex tubes and purged with oxygen (10 min). An excess of nitrogen nucleophiles (Molar ratio base/oxadiazole $=10 / 1$ ) was added and all the samples were irradiated for $3 \mathrm{~h}$. The solvent was evaporated to dryness under reduced pressure yielding a residue that was chromatographed with light petroleum/ethyl acetate at various ratios. Yields and spectroscopic data are respectively reported in Tables 1 and 2.

\section{Irradiation of 3-Phenyl-5-perfluoroheptyl-1,2,4-oxadiazole (14)}

A solution of compound (14) (0.66 g, $1.28 \mathrm{mmol})$ in dry methanol (400 mL), was partitioned into nine pyrex tubes. An excess of methylamina (Molar ratio base/oxadiazole $=10 / 1$ ) was added and all the samples were irradiated for $3 \mathrm{~h}$. Chromatography returned starting material $(0.33 \mathrm{~g} ; 50 \%)$ and gave compound (17) (0.11 g; 20\%). 
Table 2. - Physical and analytical data for 1,2,4-triazoles (8a-e)/(9a-e) and compounds (10b-d).

\begin{tabular}{|c|c|c|c|c|c|c|}
\hline Compd & $\mathrm{mp}\left({ }^{\circ} \mathrm{C}\right)$ & $\begin{array}{c}\text { IR } \\
\text { (nujol) } \\
v\left(\mathrm{~cm}^{-1}\right)\end{array}$ & $\begin{array}{c}{ }^{1} \mathrm{H} \text { NMR }(\mathrm{TMS}) \\
\delta(\mathrm{ppm})\end{array}$ & $\begin{array}{c}\mathrm{MS} \\
m / z(\%)\end{array}$ & $\begin{array}{l}\text { Molecular } \\
\text { Formula }\end{array}$ & $\begin{array}{c}\text { Analysis } \\
\text { Calcd (Found) } \\
\text { C/H/ N }\end{array}$ \\
\hline $8 a$ & $92-93^{a}$ & & $\begin{array}{l}4.10^{\mathrm{b}} \text { (s, 3H, Me), 7.54-7.56 (m, } \\
3 \mathrm{H}, \mathrm{Ar}), 7.72-7.76(\mathrm{~m}, 2 \mathrm{H}, \mathrm{Ar})\end{array}$ & $\begin{array}{l}325\left(\mathrm{M}^{+}, 100\right), 222(50), \\
194(32), 104(15), 63(17)\end{array}$ & $\mathrm{C}_{15} \mathrm{H}_{8} \mathrm{~N}_{3} \mathrm{~F}_{5}$ & $\begin{array}{l}55.39 / 2.48 / 12.92 \\
(55.20 / 2.40 / 12.80)\end{array}$ \\
\hline $8 \mathbf{b}$ & $145-147^{\mathrm{a}}$ & & $\begin{array}{l}3.96^{\mathrm{b}}(\mathrm{s}, 3 \mathrm{H}, \mathrm{Me}), 7.50-7.58(\mathrm{~m}, \\
3 \mathrm{H}, \mathrm{Ar}), 7.92-7.94(\mathrm{~m}, 1 \mathrm{H}, \mathrm{Ar}) \\
8.07-8.10(\mathrm{~m}, 2 \mathrm{H}, \mathrm{Ar})\end{array}$ & $\begin{array}{l}307\left(\mathrm{M}^{+}, 100\right), 175(8), \\
131(70), 104(37), 77(17)\end{array}$ & $\mathrm{C}_{15} \mathrm{H}_{9} \mathrm{~N}_{3} \mathrm{~F}_{4}$ & $\begin{array}{l}58.64 / 2.95 / 13.68 \\
(58.50 / 2.80 / 13.60)\end{array}$ \\
\hline 8c & $131-134^{a}$ & & $\begin{array}{l}3.91^{\mathrm{b}} \text { (s, 3H, Me), 7.05-7.30 (m, } \\
2 \mathrm{H}, \mathrm{ArF}), 7.41-7.50(\mathrm{~m}, 3 \mathrm{H}, \mathrm{Ar}) \\
8.10-8.16(\mathrm{~m}, 2 \mathrm{H}, \mathrm{Ar})\end{array}$ & $\begin{array}{l}289\left(\mathrm{M}^{+}, 100\right), 131(66) \text {, } \\
104(39), 77(14)\end{array}$ & $\mathrm{C}_{15} \mathrm{H}_{10} \mathrm{~N}_{3} \mathrm{~F}_{3}$ & $\begin{array}{l}62.28 / 3.48 / 14.53 \\
(62.10 / 3.40 / 14.40)\end{array}$ \\
\hline 8d & $62-64^{a}$ & & $\begin{array}{l}2.44^{\mathrm{b}}(\mathrm{s}, 3 \mathrm{H}, \mathrm{Me}), 3.82(\mathrm{~s}, 3 \mathrm{H} \\
\mathrm{Me}), 7.23-7.29(\mathrm{~m}, 1 \mathrm{H}, \mathrm{ArF})\end{array}$ & $\begin{array}{l}245\left(\mathrm{M}^{+}, 100\right), 70(62), 42 \\
(59)\end{array}$ & $\mathrm{C}_{10} \mathrm{H}_{7} \mathrm{~N}_{3} \mathrm{~F}_{4}$ & $\begin{array}{l}48.99 / 2.88 / 17.14 \\
(48.80 / 2.70 / 17.00)\end{array}$ \\
\hline $8 e$ & $74-76^{a}$ & & $\begin{array}{l}2.44^{\mathrm{b}} \text { (s, 3H, Me), } 3.81 \text { (s, 3H, } \\
\mathrm{Me}), 7.10-7.19(\mathrm{~m}, 1 \mathrm{H}, \mathrm{ArF}), 7.30- \\
7.34(\mathrm{~m}, 1 \mathrm{H}, \mathrm{ArF})\end{array}$ & $\begin{array}{l}227\left(\mathrm{M}^{+}, 100\right), 156(4), 69 \\
(70), 42(65))\end{array}$ & $\mathrm{C}_{10} \mathrm{H}_{8} \mathrm{~N}_{3} \mathrm{~F}_{3}$ & $\begin{array}{l}52.87 / 3.55 / 18.50 \\
(52.70 / 3.40 / 18.30)\end{array}$ \\
\hline $9 a$ & $52-53^{a}$ & & $\begin{array}{l}0.93^{\mathrm{b}}(\mathrm{t}, 3 \mathrm{H}, \mathrm{Me}, J=7 \mathrm{~Hz}), 1.98 \\
\left(\mathrm{~m}, 2 \mathrm{H}, \mathrm{CH}_{2}\right), 4.26\left(\mathrm{t}, 2 \mathrm{H}, \mathrm{CH}_{2}, J=\right. \\
7 \mathrm{~Hz}), 7.47-7.58(\mathrm{~m}, 3 \mathrm{H}, \mathrm{Ar}), 7.62- \\
7.72(\mathrm{~m}, 2 \mathrm{H}, \mathrm{Ar})\end{array}$ & $\begin{array}{l}353\left(\mathrm{M}^{+}, 100\right), 324(21) \\
310(14), 105(10)\end{array}$ & $\mathrm{C}_{17} \mathrm{H}_{12} \mathrm{~N}_{3} \mathrm{~F}_{5}$ & $\begin{array}{l}57.79 / 3.42 / 11.89 \\
(57.60 / 3.30 / 11.70)\end{array}$ \\
\hline $9 b$ & $94-97^{a}$ & & $\begin{array}{l}0.91^{\mathrm{b}}(\mathrm{t}, 3 \mathrm{H}, \mathrm{Me}, J=7 \mathrm{~Hz}), 1.98 \\
\left(\mathrm{~m}, 2 \mathrm{H}, \mathrm{CH}_{2}\right), 4.06\left(\mathrm{t}, 2 \mathrm{H}, \mathrm{CH}_{2}, J=\right. \\
7 \mathrm{~Hz}), 7.29-7.35(\mathrm{~m}, 1 \mathrm{H}, \mathrm{ArF}) \\
\text { 7.40-7.52 ( m, 3H, Ar), 8.08-8.18 } \\
\text { (m, 2H, Ar) }\end{array}$ & $\begin{array}{l}335\left(\mathrm{M}^{+}, 100\right), 306(14) \\
293(20), 104(63), 77(21)\end{array}$ & $\mathrm{C}_{17} \mathrm{H}_{13} \mathrm{~N}_{3} \mathrm{~F}_{4}$ & $\begin{array}{l}60.90 / 3.91 / 12.53 \\
(60.70 / 3.80 / 12.40)\end{array}$ \\
\hline 9c & $90-93^{a}$ & & $\begin{array}{l}0.90^{\mathrm{b}}(\mathrm{t}, 3 \mathrm{H}, \mathrm{Me}, J=7 \mathrm{~Hz}), 1.98 \\
\left(\mathrm{~m}, 2 \mathrm{H}, \mathrm{CH}_{2}\right), 4.05\left(\mathrm{t}, 2 \mathrm{H}, \mathrm{CH}_{2}, J=\right. \\
7 \mathrm{~Hz}), 7.10-7.22(\mathrm{~m}, 2 \mathrm{H}, \mathrm{ArF}) \\
\text { 7.32-7.48 ( m, 3H, Ar), 8.12-8.16 } \\
\text { (m, 2H, Ar) }\end{array}$ & $\begin{array}{l}317\left(\mathrm{M}^{+}, 100\right), 289(10), \\
131(23), 104(62), 77(17)\end{array}$ & $\mathrm{C}_{17} \mathrm{H}_{14} \mathrm{~N}_{3} \mathrm{~F}_{3}$ & $\begin{array}{l}64.35 / 4.45 / 13.24 \\
(64.20 / 4.30 / 13.10)\end{array}$ \\
\hline 9d & Oil & & $\begin{array}{l}0.84^{\mathrm{b}}(\mathrm{t}, 3 \mathrm{H}, \mathrm{Me}, J=7 \mathrm{~Hz}), 1.84 \\
\left(\mathrm{~m}, 2 \mathrm{H}, \mathrm{CH}_{2}\right), 2.42(\mathrm{~s}, 3 \mathrm{H}, \mathrm{Me}) \\
3.93\left(\mathrm{t}, 2 \mathrm{H}, \mathrm{CH}_{2}, J=7 \mathrm{~Hz}\right), 7.15- \\
7.27(\mathrm{~m}, 1 \mathrm{H}, \mathrm{ArF})\end{array}$ & $\begin{array}{l}273\left(\mathrm{M}^{+}, 100\right), 244(29) \\
231(48), 190(40), 69(44)\end{array}$ & $\mathrm{C}_{12} \mathrm{H}_{11} \mathrm{~N}_{3} \mathrm{~F}_{4}$ & $\begin{array}{l}52.75 / 4.06 / 15.38 \\
(52.60 / 3.90 / 15.20)\end{array}$ \\
\hline $9 e$ & Oil & & $\begin{array}{l}0.84^{\mathrm{b}}(\mathrm{t}, 3 \mathrm{H}, \mathrm{Me}, J=7 \mathrm{~Hz}), 1.88 \\
\left(\mathrm{~m}, 2 \mathrm{H}, \mathrm{CH}_{2}\right), 2.44(\mathrm{~s}, 3 \mathrm{H}, \mathrm{Me}) \\
3.94\left(\mathrm{t}, 2 \mathrm{H}, \mathrm{CH}_{2}, J=7 \mathrm{~Hz}\right), 7.11- \\
7.18(\mathrm{~m}, 2 \mathrm{H}, \mathrm{ArF})\end{array}$ & $\begin{array}{l}255\left(\mathrm{M}^{+}, 100\right), 226(21) \\
213(43), 172(39), 69(56)\end{array}$ & $\mathrm{C}_{12} \mathrm{H}_{12} \mathrm{~N}_{3} \mathrm{~F}_{3}$ & $\begin{array}{l}56.47 / 4.74 / 16.46 \\
(56.40 / 4.70 / 16.40)\end{array}$ \\
\hline $10 \mathrm{~b}$ & $108-110^{a}$ & $\begin{array}{l}3310 \\
1670\end{array}$ & $\begin{array}{l}3.89^{\mathrm{C}}(\mathrm{s}, 3 \mathrm{H}, \mathrm{MeO}), 7.45-7.50 \text { (m, } \\
3 \mathrm{H}, \mathrm{Ar}), 7.63-7.69(\mathrm{~m}, 2 \mathrm{H}, \mathrm{Ar}) \\
7.80-7.84(\mathrm{~m}, 1 \mathrm{H}, \mathrm{ArF}), 10.55^{\mathrm{d}}(\mathrm{s}, \\
1 \mathrm{H}, \mathrm{NH})\end{array}$ & $\begin{array}{l}326\left(\mathrm{M}^{+}, 25\right), 295(93) \\
177(100), 149(35), 104 \\
(44), 77(25)\end{array}$ & $\mathrm{C}_{15} \mathrm{H}_{10} \mathrm{~N}_{2} \mathrm{O}_{2} \mathrm{~F}_{4}$ & $\begin{array}{l}55.22 / 3.09 / 8.59 \\
(55.10 / 3.00 / 8.50)\end{array}$ \\
\hline 10c & $143-144^{a}$ & $\begin{array}{l}3240 \\
1650\end{array}$ & $\begin{array}{l}3.97^{\mathrm{c}}(\mathrm{s}, 3 \mathrm{H}, \mathrm{MeO}), 7.47-7.49(\mathrm{~m}, \\
3 \mathrm{H}, \mathrm{Ar}), 7.53-7.59(\mathrm{~m}, 2 \mathrm{H}, \mathrm{ArF}) \\
7.61-7.65(\mathrm{~m}, 2 \mathrm{H}, \mathrm{Ar}), 10.53^{\mathrm{d}} \text { (s, } \\
1 \mathrm{H}, \mathrm{NH})\end{array}$ & $\begin{array}{l}308\left(\mathrm{M}^{+}, 20\right), 277(51), \\
159(100), 131(29), 104 \\
(21), 77(22)\end{array}$ & $\mathrm{C}_{15} \mathrm{H}_{11} \mathrm{~N}_{2} \mathrm{O}_{2} \mathrm{~F}_{3}$ & $\begin{array}{l}58.45 / 3.60 / 9.09 \\
(58.30 / 3.50 / 9.00)\end{array}$ \\
\hline 10d & $97-99^{\mathrm{a}}$ & $\begin{array}{l}3400 \\
1680\end{array}$ & $\begin{array}{l}2.29^{\mathrm{c}}(\mathrm{s}, 3 \mathrm{H}, \mathrm{Me}), 3.81(\mathrm{~s}, 3 \mathrm{H} \\
\mathrm{MeO}), 7.72-7.83(\mathrm{~m}, 1 \mathrm{H}, \mathrm{ArF}) \\
\left.10.17^{\mathrm{d}} \text { (s, } 1 \mathrm{H}, \mathrm{NH}\right)\end{array}$ & $\begin{array}{l}264\left(\mathrm{M}^{+}, 33\right), 233(58), \\
177(100), 149(29),\end{array}$ & $\mathrm{C}_{10} \mathrm{H}_{8} \mathrm{~N}_{2} \mathrm{O}_{2} \mathrm{~F}_{4}$ & $\begin{array}{l}45.46 / 3.05 / 10.60 \\
(45.30 / 2.90 / 10.40)\end{array}$ \\
\hline
\end{tabular}

\footnotetext{
${ }^{\text {a }}$ Crystallization solvent: Light petroleum

$\mathrm{b}$ in $\mathrm{CDCl}_{3}$

c in DMSO-d 6

d exchangeable with $\mathrm{D}_{2} \mathrm{O}$
} 


\section{ACKNOWLEDGEMENT}

Financial support through the Italian MIUR and University of Palermo within the National Research Projects "Fluorinated Compounds: New Materials for Advanced Applications" (PRIN 2001) and "Fluorinated Nanoreactors with Designed Structures and Optimised Functions” (PRIN 2003) is gratefully acknowledged.

\section{REFERENCES}

1 (a) K. Burger, U. Wucherpfennig, and E. Brunner, Adv. Heterocycl. Chem., 1994, 60, 1, and references cited therein; (b) E. Differding, W. Frick, R. W. Lang, P. Martin, C. Schmit, S. Veenstra, and H. Greuter, Bull. Soc. Chim. Belg., 1990, 99, 647; (c) J. C. Sloop, C. L. Bumgardner, and W. D. Loehle., J. Fluorine Chem., 2002, 118,135; (d) S. Z. Zhu, Y. L. Wang, W. M. Peng, L. P. Song, and G. F. Jin, Curr. Org. Chem., 2002, 6, 1057.

(a) R. D. Chambers and C. R Sargent, Adv. Heterocycl. Chem., 1981, 28, 1; (b) M. J. Silvester, Adv. Heterocycl. Chem., 1994, 59, 1; (c) G. G. Furin, Syntheses of heterocyclic compounds with perfluoroalkyl groups based on internal perfluoroolefins, in: Targets in Heterocyclic Systems, ed. by O. A. Attanasi and D. Spinelli, Vol. 2, Societa Chimica Italiana, Roma, 1998, pp. 355-441.

$3 \quad$ N. Vivona and S. Buscemi, Heterocycles, 1995, 41, 2095.

4 N. Vivona, S. Buscemi, V. Frenna, and G. Cusmano, Adv. Heterocycl. Chem., 1993, 56, 49.

5 (a) S. Buscemi, A. Pace, and A. Vivona, Tetrahedron Lett., 2000, 41, 7977; (b) S. Buscemi, A. Pace, R. Calabrese, N. Vivona, and P. Metrangolo, Tetrahedron, 2001, 57, 5865; (c) S. Buscemi, A. Pace, I. Pibiri, N. Vivona, and T. Caronna, J. Fluorine Chem., 2004, 125, 165.

A. Pace, I. Pibiri, S. Buscemi, N. Vivona, and L. Malpezzi, J. Org. Chem., 2004, 69, 4108.

7

S. Buscemi, A. Pace, A. Palumbo Piccionello, I. Pibiri, and N. Vivona, Heterocycles, 2004, 63, 1619.

8 J. H. Lange, C. G. Kruse, A. C. McCreary, and H. H. Van Stuivenberg, PCT Int. Appl. 2004 (Chem. Abstr., 2004, 140, 303680).

9 H. Maezaki, T. Ichikawa, and K. Ikedo, Jpn. Kokai Tokkyo Koho 2003 (Chem. Abstr., 2003, 139, 375025).

10 J. B. Camden, U.S. Pat. Appl. Publ. 2001, (Chem. Abstr., 2001, 135, 339239).

11 (a) H. Xue, B. Twamley, and J. M. Shreeve, J. Org. Chem., 2004, 69, 1397; (b) Y. R. Mirzaei, B. Twamley, and J. M. Shreeve, J. Org. Chem., 2002, 67, 934.

12 M. Schmutz, PCT Int. Appl. 2000, (Chem. Abstr., 2000, 133, 213182).

13 B. T. Kim, S. Y. Han, and C. S. Pak, PCT Int. Appl. 2000, (Chem. Abstr., 2000, 133, 120337). 
14 (a) J. Ehrenfreund, H. Tobler, and H. Walter, PCT Int. Appl. 2004, (Chem. Abstr., 2004, 140, 235719); (b) M. Schmutz, U.S. Patent 2000, (Chem. Abstr., 2000, 132, 260713); (c) S. Takeda, Y. Kaneko, M. Tokizawa, H. Eto, K. Ishida, K. Maebashi, M. Matsumoto, T. Asaoka, and S. Sato, Eur. Pat. Appl. 1999, (Chem. Abstr,, 1999, 131, 73657); (d) T. Kagiya, M. Abe, S. Nishimoto, Y. Shibamoto, K. Shimokawa, Y. Hisanaga, T. Nakada, and T. Yoshizawa, Eur. Pat. Appl. 1988, (Chem. Abstr., 1988, 111, 7409).

15 K. Funabiki, N. Noma, G. Kuzuya, M. Matsui, and K. Shibata, J. Chem. Res., 1999, (S) 300; (M) 1301, and references therein for recent literature on fluorinated 1,2,4-triazoles.

16 S. Buscemi, A. Pace, I. Pibiri, N. Vivona, and D. Spinelli, J. Org. Chem., 2003, 68, 605.

17 S. Buscemi, N. Vivona, and T. Caronna, J. Org. Chem., 1996, 61, 8397.

18 S. Buscemi, A. Pace, N. Vivona, T. Caronna, and A. Galia, J. Org. Chem., 1999, 64, 7028.

19 S. Buscemi, A. Pace, I. Pibiri, N. Vivona, C. Z. Lanza, and D. Spinelli, Eur. J. Org. Chem., 2004, 974.

20 J. B. Polya, In Comprehensive Heterocyclic Chemistry, ed. by A. R. Katritzky and C. W. Rees, Pergamon, Elsevier Science, 1984, Vol. 5, pp. 733-790.

21 G. S. Shchegoleva, V. A. Barkhash, and N. N. Vorozhtsov Jr., Izv. Akad. Nauk SSSR, Ser. Khim.,1967, 3, 708 (Chem. Abstr., 1967, 67, 100075).

22 J. P. Critchley and J. S. Pippett, J. Fluorine Chem., 1973, 2, 13.

23 L. J. Belf, M. W. Buxton, and J F. Tilney-Bassett, Tetrahedron, 1967, 23, 4719.

24 C. Kimura, K. Kashiwaya, M. Kobayashi, and T. Nishiyama, J. Am. Oil Chem. Soc., 1984, 69,105 (Chem. Abstr., 1984, 100, 158577). 